\title{
Antiretroviral Drug Treatment of Individuals that Used Preexposure Prophylaxis (PrEP) Before Diagnosis
}

\author{
David A. M. C. van de Vijver, PharmD PhD ${ }^{*}$ \\ Shreoshee Mukherjee, MPP \\ Jeroen J.A. van Kampen, MD, PhD
}

\author{
Address \\ "Viroscience Department, Erasmus Medical Centre, Rotterdam, The Netherlands \\ Email: d.vandevijver@erasmusmc.nl
}

Published online: 8 March 2021

(C) The Author(s) 2021

This article is part of the Topical Collection on HIV Medicine

Recommendations

- First-line antiretroviral drug treatment of individuals that used PrEP before diagnosis should be optimized based on a genotypic resistance test.

- Although there are no systematic studies on the optimal treatment of individuals in whom drug resistance due to PrEP is found, there is anecdotal evidence that the emtricitabine-associated mutation M184I/N and/or the tenofovir-associated mutation (K65R) can be treated with currently recommended first-line regimens including dolutegravir-based, bictegravir-based, or darunavir-based regimens boosted with tenofovir plus emtricitabine or lamivudine.

- Due to a lack of data, induction treatment using dual therapy including dolutegravir and lamivudine is not recommended. Maintenance treatment with dual therapy can be considered after achieving viral suppression.

Keywords HIV · PrEP • Drug resistance · Antiretroviral drug treatment · M184I/V • K65R

\begin{abstract}
Purpose of review The antiretroviral drugs, tenofovir and emtricitabine used as preexposure prophylaxis (PrEP), are also used in treatment of HIV. Drug resistance due to PrEP can therefore jeopardize future treatment options. This review discusses treatment of individuals that used PrEP in whom viral mutations against tenofovir (K65R) or emtricitabine (M184I/V) are found.

Recent findings Although no studies systematically investigated the optimal treatment of individuals who used PrEP before diagnosis, there is anecdotal evidence that HIV including the $\mathrm{K} 65 \mathrm{R}$ and/or M184I/V can be successfully treated using recommended first-line regimens.

Summary Drug resistance can be ascribed to use of PrEP while having an unrecognized acute HIV infection, partial adherence to PrEP, and transmission of HIV resistant to PrEP drugs. First-line antiretroviral drug treatment in individuals who used PrEP before diagnosis must be optimized based on genotypic resistance test results. Individuals in whom M184I/V and/or K65R is detected can be treated with dolutegravir-based, bictegravir-
\end{abstract}


based, or darunavir-based regimens plus tenofovir plus lamivudine or emtricitabine. Dual therapy using dolutegravir plus lamivudine is not recommended for induction therapy in individuals with viral mutations against the drugs used as PrEP. There is an urgent need to confirm the anecdotal evidence for successful treatment using first-line regimens.

\section{Introduction}

The continuous development of novel antiretroviral drugs has resulted in significant improvements in the treatment and prevention of HIV. Due to these improvements, individuals living with HIV can have a nearnormal life span when they adhere to antiretroviral drug treatment [1]. Antiretroviral drugs can be used for prevention of HIV transmission as individuals living with HIV that are virally suppressed due to antiretroviral drug treatment cannot transmit the virus to others [2, 3]. Another important antiretroviral drug-based strategy for prevention of HIV transmission is the use of preexposure prophylaxis (PrEP) with the antiretroviral drugs tenofovir alone or in combination with emtricitabine (FTC) in individuals at high risk of infection [4]. (Tenofovir is given as the prodrug tenofovir disoproxil fumarate-TDF, or tenofovir alafenamide fumarate-TAF.) Based on these breakthroughs, the World Health Organization (WHO) has declared ending the AIDS pandemic as one of its sustainable development goals in 2030 which aim to achieve a better and sustainable future for all people across the World [5].

To reach the ambitious goal of ending the AIDS pandemic, the WHO recommends a comprehensive package of prevention strategies including condoms, counselling, male circumcision [6, 7], and HIV testing followed by immediate antiretroviral drug treatment in those testing positive $[2,3]$. The WHO also recommends that people with a substantial risk of HIV infection are offered PrEP [8]. Importantly, the PrEP drugs tenofovir and emtricitabine are frequently prescribed for treatment of HIV, and drug resistance arising due to the use of PrEP can therefore jeopardize future treatment options [9].

The aim of this review is to discuss antiretroviral drug treatment in individuals that used PrEP before diagnosis. To address this aim, we will first describe the mutations that are involved in drug resistance to the PrEP drugs tenofovir and FTC. We will then discuss the processes by which drug resistance can emerge and the drug resistance-associated mutations that have been reported in randomized controlled trials on the efficacy of PrEP for prevention of HIV transmission and in real-world settings. We will then discuss diagnosis and treatment of drug resistance in individuals who had used PrEP before diagnosis.

\section{Resistance-Associated Mutations to Tenofovir and Emtricitabine}

Drug resistance to the PrEP drugs tenofovir and FTC is well-characterized. Only a single point mutation in the reverse transcriptase gene of the viral genome is required for resistance to tenofovir (K65R). Additionally, drug resistance to tenofovir can occur in HIV that has accumulated multiple thymidine associated mutations (TAMs), a complex set of mutations associated with resistance to thymidine analogous, such as zidovudine. In particular, the response to tenofovir is reduced if at least three different TAMs are present including M41L or L210W (the other TAMs are D67N, K70R, T215F/Y, and K219Q/E/ $\mathrm{R} / \mathrm{N})$ [10]. 
Resistance to FTC is encoded by a single point mutation in reverse transcriptase (M184V) [11, 12]. In patients developing resistance to emtricitabine, the M184I mutation usually emerges before M184V [13-20, 21••].

Cross-resistance between the drugs used as PrEP is limited as HIV including the hallmark FTC mutation M184I/V remains susceptible to tenofovir. Similarly, HIV containing multiple TAMs remains susceptible to FTC. K65R has been reported to result in intermediate resistance to emtricitabine. Irrespective of cross-resistance, it is important to note that viruses harboring both the K65R and M184V/I mutations are rarely detected. Instead, K65R and M184V/I are frequently found as single mutations on different genomes [22].

\section{Processes by Which Drug Resistance Jeopardizes PrEP}

Epidemiological studies from randomized controlled trials that studied the efficacy of PrEP (Table 1) [13-20, 21••] and from real-world settings (Table 2) $[24,25 \bullet, 26]$ have identified several processes by which drug resistance jeopardizes PrEP. These processes include the rapid emergence of resistance while using PrEP during an unrecognized acute infection [13-20, 21••], the emergence of drug resistance in individuals that are partially adherent to PrEP [18], and transmission of drug-resistant HIV to individuals using PrEP [24, $25 \bullet, 26]$. The three processes will be discussed in further detail in the following paragraphs.

Start of PrEP During an Unrecognized Acute Infection

PrEP should only be started after a negative HIV test as the genetic barrier for resistance, defined as the number of mutations required to overcome drug selected pressure $[11,27]$, is too low for tenofovir and FTC. Consequently, resistance can emerge in individuals using tenofovir and FTC as their only antiretroviral drugs. Unfortunately, HIV tests cannot identify very acute infections in the time period between infection and when the HIV test can reliably detect the infection (window period ) [28]. Individuals who test negative for HIV during this window period, which depending on the HIV test is at least 1012 days after exposure [28], can have an unrecognized acute HIV infection at the

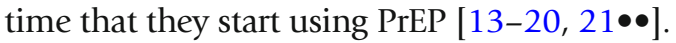

The randomized controlled trials showed that the few individuals that started PrEP while having an unrecognized HIV infection [13-20, 21 ••] have a substantially high risk for drug resistance. Among 35 individuals randomized to receive TDF/FTC or TDF as PrEP during an unrecognized HIV infection, drug resistance was later found in nine individuals $(26 \%)$. The high risk of drug resistance in individuals with an unrecognized acute infection can be explained by the high viral replication rate during the acute stage and high viral mutation rate of HIV [29] which can result in the rapid selection of drug resistance associated mutations.

The most commonly found drug resistance-associated mutation was M184I/ $\mathrm{V}$ which was detected in seven out of nine individuals who had started PrEP at the time when they had an unrecognized acute infection (78\%) [13-20, 21••]. K65R was reported in two individuals, of whom one person used TDF as the single PrEP drug [14]. The second person in whom K65R was found used TDF/ 


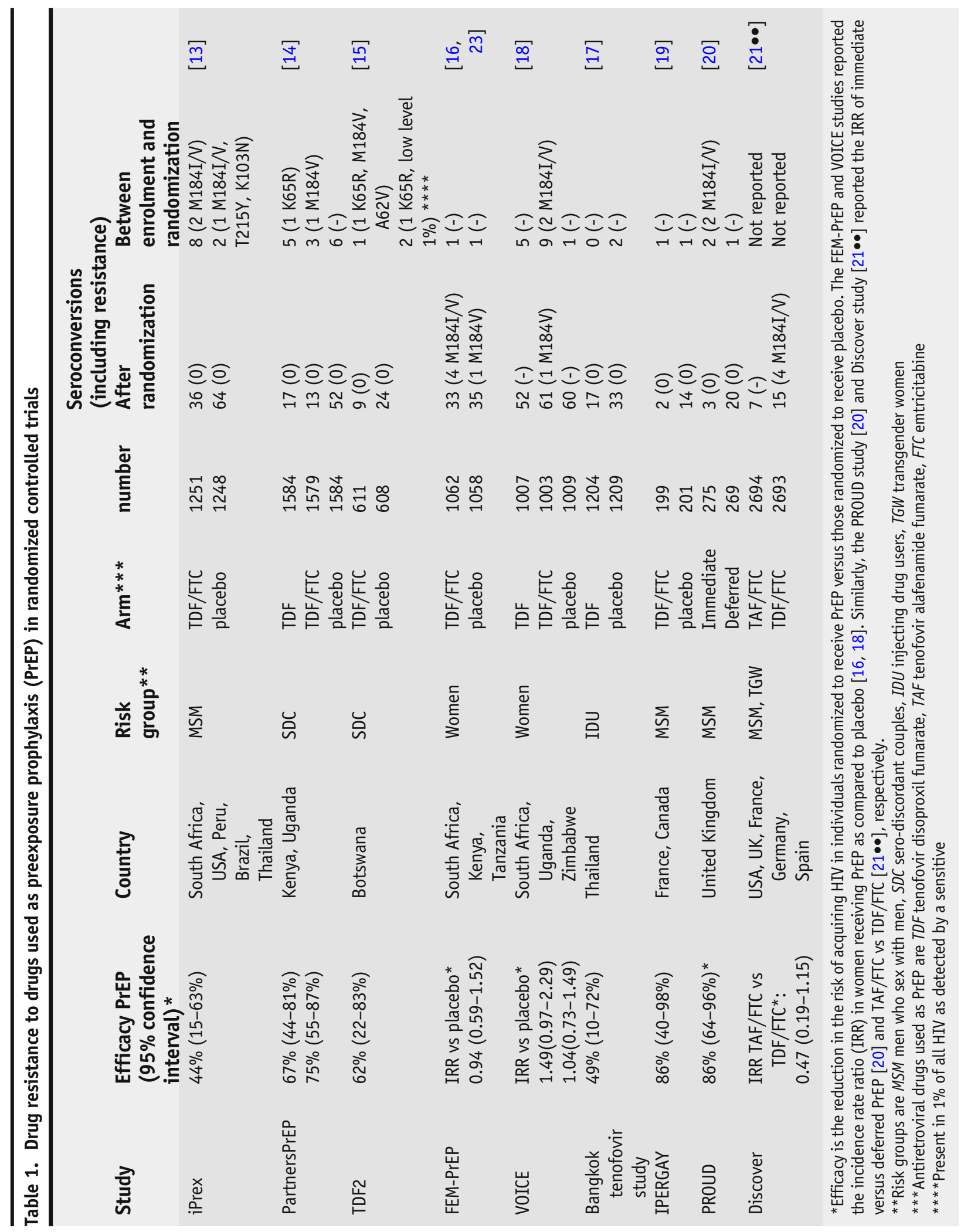




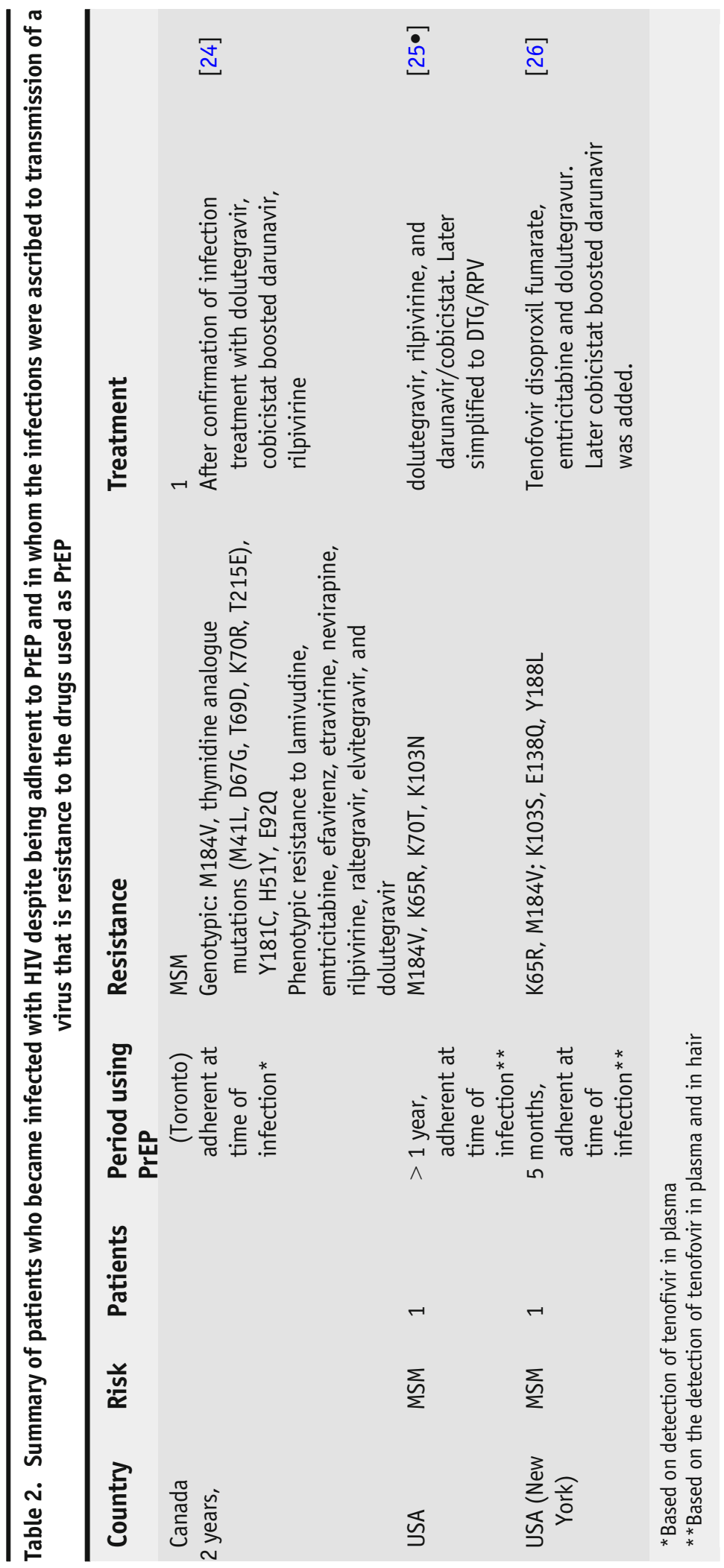


FTC and came from Botswana [15], where HIV is predominantly of subtype C. K65R emerges more rapidly in subtype $\mathrm{C}$ viruses [30], which has been ascribed to the unique subtype $\mathrm{C}$ sequence context in the region of K65R. Specifically, subtype $\mathrm{C}$ includes a span of five consecutive adenosines preceding the adenosine at the second position in the K65 codon rendering it more likely to be mutated during reverse transcription [31].

\section{Emergence of Drug Resistance in Individuals that Are Partially Adherent to PrEP}

The emergence of drug resistance after randomization is reported by three randomized controlled trials $[16,18,21 \bullet \bullet]$, of which two studies did not find a HIV preventative benefit of PrEP [16, 18] (Table 1). Two randomized controlled trials, FEM-PrEP and Discover $[16,21 \bullet \bullet]$, reported that patients were found to be infected early during the study. Because FEM-PrEP and Discover did not systematically investigate if individuals had seroconverted between the time of HIV testing and the start of PrEP and consequently could not rule out that people had started using PrEP while having an unrecognized acute infection $[16,21 \bullet \bullet]$. The third study reported poor adherence to PrEP based on the detection of tenofovir-plasma levels above a particular threshold which indicated the recent use of PrEP. The emergence of resistance in this third study may therefore be explained by (partial) non-adherence allowing HIV to select for drug resistance-associated mutations after infection [16, 18].

\section{Transmission of Drug Resistance}

Drug resistance can jeopardize the preventative benefits of PrEP when an individual becomes infected with a virus that is resistant to the drugs used as PrEP. Three patients have been reported who became infected with a drugresistant strain of HIV despite being consistently adherent to $\operatorname{PrEP}[24,25 \bullet, 26]$, as confirmed by detection of tenofovir in plasma $[24,25 \bullet, 26]$ or in hair of the individuals $[24,26]$ (Table 2). All three patients were infected with a multidrug-resistant virus which not only included resistance associated mutations to emtricitabine, M184I/V [24, 25•, 26], and tenofovir (K65R in two individuals $[25 \bullet, 26]$, and a combination of TAMs in one patient [24]) but also to nonnucleoside reverse transcriptase inhibitors $[24,25 \bullet, 26]$ and in one individual also to integrase inhibitors [24].

\section{Risk of Resistance in Real-World Settings}

During recent years, two studies reported M184I/V in 23\% (out of 91 individ-

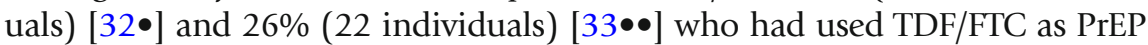
before diagnosis $[32 \bullet, 33 \bullet \bullet$. The studies did not find K65R in any of the individuals who had used PrEP. The high risk of resistance could be ascribed to the use of PrEP after being infected with HIV, as indicated by the observation that one-third of patients in one study were in the acute stage of infection [32•]. Similarly, the second study reported a strongly reduced HIV-1 RNA load at the

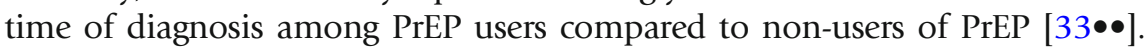
Such low level of HIV-1 RNA among one group could indicate the continued use of PrEP at the time of infection or a blunted viremia during breakthrough infections with PrEP [34]. 


\section{Treatment of HIV-Infected Individuals that Used PrEP Before Diagnosis}

Although the emergence of resistance has been recognized as a limitation of the use of PrEP, there are no reports that systematically studied the optimal antiretroviral drug treatment regimen for individuals who have been exposed to PrEP before HIV diagnosis. Considering the relatively high rate of drug resistance mutations detected in patients who fail PrEP, first-line antiretroviral drug treatment must be optimized based on the genotypic resistance test results [35•]. Treatment optimization based on a genotypic resistance test resulted in virological suppression in the three patients presented in Table 2 that became infected due to transmission of HIV that had drug resistance-associated mutations to tenofovir and emtricitabine $[24,25 \bullet, 26]$. In particular, all three patients were treated successfully with dolutegravir plus cobicistat-boosted darunavir $[24,25 \bullet$, $26]$, in combination with rilpivirine $[24,26]$ or TDF/FTC [25•].

In the following paragraphs, we will discuss the treatment options for the most frequently reported mutations associated with resistance to PrEP. We will follow the preferred initial antiretroviral drug regimens of the International Antiviral Society-USA which recommends to start treatment with any of the following combinations: bictegravir plus TAF plus FTC, dolutegravir plus TAF or TDF plus FTC/lamivudine, or dolutegravir plus lamivudine [35•].

A key challenge in the detection of M184I/V is that these mutations rapidly revert to a drug susceptible wild-type virus if PrEP is discontinued [36]. Importantly, after reversion, drug resistance-associated mutations can still be detected in latently infected long-lived reservoir cells. Consequently, a genotypic resistance test may not identify M184I/V in plasma, but this drug-resistant strain can re-emerge by stochastic reactivation of drug-resistant proviruses from the reservoir [37].

The M184I/V mutation does not only result in resistance to emtricitabine but also to lamivudine $[38,39]$ and to abacavir [40]. Importantly, the presence of $\mathrm{M} 184 \mathrm{I} / \mathrm{V}$ is not a contraindication to combination treatment including FTC or lamivudine. M184I/V is associated with lower viral replication resulting in reduced HIV-1 RNA load [38, 39], and which in turn reduces the risk of emergence of AIDSrelated morbidity and mortality [41]. In addition, M184I/V also increases the susceptibility of HIV to tenofovir [42]. There exists some evidence that patients with the M184I/V viral mutation, ascribed to the use of PrEP before diagnosis, can indeed

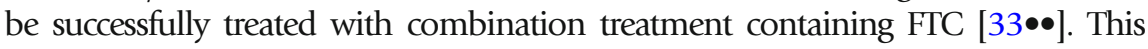
limited evidence comes from four patients who had undetectable HIV viral load 3 months into treatment despite the presence of the M184I/V mutation ascribed to the use of PrEP before diagnosis, after treatment with a regimen including emtricitabine (in addition to FTC, two patients were also treated with the recommended initial regimen TDF and dolutegravir [35•]; and two were also treated with TDF and

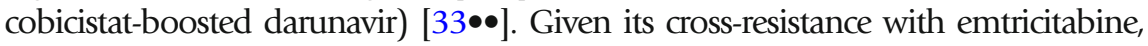
it is likely that $\mathrm{M} 184 \mathrm{I} / \mathrm{V}$ can also be successfully treated with lamivudine, which is recommended in initial regimens [35•]. The limited impact of $\mathrm{M} 184 \mathrm{I} / \mathrm{V}$ is also reported in clinical studies that report that patients with an archived M184V/I 
mutation detected by proviral DNA genotyping can successfully be treated with bictegravir or dolutegravir plus TAF/FTC or dolutegravir plus abacavir plus lamivudine [35•, 43-45]. In addition, the DAWNING study showed that patients who fail first-line antiretroviral drug treatment with emergence of the M184I/V mutation in plasma can be successfully treated with dolutegravir combined with 2 nucleoside reverse transcriptase inhibitors [46].

Simplifying initial three drug antiretroviral drug regimens to two drug regimens has been documented to maintain viral suppression in individuals without prior virological failure or evidence of drug resistance $[35 \bullet$, 47]. Recent studies have reported that patients who had the M184I/V viral mutation before viral suppression can be successfully switched to a two drug regimen including lamivudine $[48,49]$. It should be noted that all these studies are on maintenance therapy; i.e., patients who were already virologically suppressed with combination antiretroviral drug therapy consisting of at least three different antiretroviral drugs were switched to dual therapy. There is currently no data published on the virological suppression rates in patients who start dual therapy (induction therapy to achieve virological suppression immediately after diagnosis) while being infected with a HIV strain harboring M184I/V mutation or who failed PrEP. Thus, the recommended first-line dual therapy with dolutegravir and emtricitabine cannot be recommended yet in patients who failed PrEP [35•].

\section{K65R}

The K65R viral mutation reduces the susceptibility to all nucleoside reverse transcriptase inhibitors except zidovudine. Treatment with zidovudine is, however, not recommended because of high rates of serious toxicities, including peripheral neuropathy and mitochondrial toxicity that may lead to myopathy, hepatic steatosis, lactic acidosis, lipoatrophy, and bone marrow suppression [50]. Contrary to the frequently reported M184I/V mutation [51], K65R is rarely reported [52]. Consequently, there are few studies that systematically investigated the risk of K65R on future virological failure. One study including a small number of patients suggested that bictegravir plus TAF/FTC may be effective when the K65R mutation is present $[35 \bullet, 44]$. There are no studies on the impact of K65R on regimens that include TDF.

Despite of the limited information on the impact of K65R that is available, the International Antiviral Society-USA for antiretroviral drug treatment of HIV recommends that dolutegravir-based, bictegravir-based, or darunavir-based regimens boosted with TDF or TAF plus FTC or lamivudine would still be expected to achieve high rates of viral suppression even in the presence of K65R and/or M184I/V [35•] and can be subsequently tailored according to clinical resistance test results.

There have been no systematic studies on maintenance therapy or on induction therapy with dual therapy in the presence of the K65R mutation. Thus, evidence is currently lacking to recommend dual therapy in PrEP failures. Anecdotal evidence from a single patient that became infected with PrEPresistant virus including K65R and M184V found that treatment could be successfully simplified to dolutegravir and rilpivirine after achieving viral suppression [25•]. Induction treatment with dual therapy in individuals with the K65R viral mutation cannot be recommended. 


\section{Discussion}

\section{Conclusion}

In this review, we have shown that drug resistance due to PrEP can emerge due to three different processes including the start of PrEP, while having an unrecognized acute infection, partial adherence after infection with HIV, and transmission of a virus that is resistant to the drugs used as PrEP. If drug resistance arises, then it usually involves the M184I/V viral mutation and to a lesser extent the K65R viral mutation. It should, however, be noted that K65R could emerge more frequently in subtype $\mathrm{C}$ as this clade more rapidly for K65R as compared to other subtypes [30, 31].

The treatment regimen in individuals that used PrEP before diagnosis should be adjusted based on the genotypic resistance results before start of antiretroviral drug treatment [35•]. Adjustment of treatment is especially important in PrEP using individuals that became infected with a virus that is resistant to the drugs used as PrEP $[24,25 \bullet$, 26]. Although no studies have investigated the optimal treatment regimen of individuals that used PrEP before HIV diagnosis, there is limited evidence that patients infected with a M184I/V and/or K65R viral mutation can be treated with a preferred initial regimen of a second-generation integrase inhibitor (bictegravir or dolutegravir) and two nucleoside reverse transcriptase inhibitors as recommended by the International Antiviral Society-USA. Importantly, before any strong recommendations can be made, it is important that this limited impact on future treatment options is confirmed in a clinical study including individuals that used PrEP before diagnosis. Simplification of treatment to a two-drug regimen seems possible in those virologically suppressed individuals that had the M184V viral mutation before start of treatment, but not for induction therapy.

This review was limited to the currently available PrEP agents including TAF/ FTC, TDF and TDF/FTC. In future years, novel antiretroviral agents that can be used as PrEP are expected to become available. These novel PrEP drugs include long-acting agents including the integrase inhibitor cabotegravir [53] and a novel reverse transcriptase translocation inhibitor islatravir [54]. Cabotegravir has cross-resistance to all licensed integrase inhibitors [55]. Resistance to islatravir involves the M184V viral mutation [54].

In conclusion, drug resistance can emerge in individuals who use PrEP. Although the evidence that is available suggests that drug resistance due to PrEP usually does not have a profound impact on future treatment options, the antiretroviral drug regimen should be adjusted using a genotypic resistance test in patients that used PrEP before diagnosis. There is an urgent need for clinical studies that investigate the optimal treatment of individuals who were PrEP users before diagnosis of HIV. These studies should also be performed in areas in where subtype $\mathrm{C}$ is common, given the rapid selection of $\mathrm{K} 65 \mathrm{R}$ in this clade [30, 31]. 


\section{Funding}

Research reported in this publication was supported by the National Institute Of Allergy And Infectious Diseases of the National Institutes of Health under Award Number R01AI147330. The content is solely the responsibility of the authors and does not necessarily represent the official views of the National Institutes of Health. Dr. van de Vijver reports grants from Gilead Sciences, grants from ViiV, grants from MSD, outside the submitted work.

\section{Declarations}

\section{Conflicts of interest}

Drs Mukherjee and van Kampen do not report any conflict of interest.

Open Access This article is licensed under a Creative Commons Attribution 4.0 International License, which permits use, sharing, adaptation, distribution and reproduction in any medium or format, as long as you give appropriate credit to the original author(s) and the source, provide a link to the Creative Commons licence, and indicate if changes were made. The images or other third party material in this article are included in the article's Creative Commons licence, unless indicated otherwise in a credit line to the material. If material is not included in the article's Creative Commons licence and your intended use is not permitted by statutory regulation or exceeds the permitted use, you will need to obtain permission directly from the copyright holder. To view a copy of this licence, visit http://creativecommons.org/licenses/by/4.0/.

\section{References and Recommended Reading}

Papers of particular interest, published recently, have been highlighted as:

- Of importance

$\bullet \quad$ Of major importance

1. Mills EJ, Bakanda C, Birungi J, Chan K, Ford N, Cooper $\mathrm{CL}$, et al. Life expectancy of persons receiving combination antiretroviral therapy in low-income countries: a cohort analysis from Uganda. Ann Intern Med. 2011;155(4):209-16.

2. Rodger AJ, Cambiano V, Bruun T, Vernazza P, Collins $\mathrm{S}$, Degen $\mathrm{O}$, et al. Risk of HIV transmission through condomless sex in serodifferent gay couples with the HIV-positive partner taking suppressive antiretroviral therapy (PARTNER): final results of a multicentre, prospective, observational study. Lancet. 2019;393(10189):2428-38.

3. Cohen MS, Chen YQ, McCauley M, Gamble T, Hosseinipour MC, Kumarasamy N, et al. Antiretroviral therapy for the prevention of HIV-1 transmission. N Engl J Med. 2016;375:830-9.

4. Riddell Jt AKR, Mayer KHHIV. Preexposure prophylaxis: a review. Jama. 2018;319(12):1261-8.

5. World Health Organization. Global health sector strategy on HIV, 2016-2021. Geneva: WHO; 2016.

6. Bailey RC, Moses S, Parker CB, Agot K, Maclean I, Krieger JN, et al. Male circumcision for HIV prevention in young men in Kisumu, Kenya: a randomised controlled trial. Lancet. 2007;369(9562):643-56.

7. Gray RH, Kigozi G, Serwadda D, Makumbi F, Watya S, Nalugoda F, et al. Male circumcision for HIV prevention in men in Rakai, Uganda: a randomised trial. Lancet. 2007;369(9562):657-66.

8. World Health Organization. Consolidated guidelines on the use of antiretroviral drugs for treating and preventing HIV infections. Recommendation for a public health approach Geneva 20162016.

9. Dimitrov DT, Boily MC, Hallett TB, Albert J, Boucher C, Mellors JW, et al. How much do we know about drug resistance due to prep use? analysis of experts' opinion and its influence on the projected public health impact. PLoS One. 2016;11(7):e0158620.

10. Miller MD. K65R, TAMs and tenofovir. AIDS Rev. 2004;6(1):22-33.

11. van de Vijver DA, Boucher CA. The risk of HIV drug resistance following implementation of pre-exposure prophylaxis. Curr Opin Infect Dis. 2010;23(6):621-7.

12. van de Vijver DA, Nichols BE, Abbas UL, Boucher CA, Cambiano V, Eaton JW, et al. Preexposure prophylaxis will have a limited impact on HIV-1 drug resistance in 
sub-Saharan Africa: a comparison of mathematical models. Aids. 2013;27(18):2943-51.

13. Grant RM, Lama JR, Anderson PL, McMahan V, Liu AY, Vargas L, et al. Preexposure chemoprophylaxis for HIV prevention in men who have sex with men. N Engl J Med. 2010;363(27):2587-99.

14. Baeten JM, Donnell D, Ndase P, Mugo NR, Campbell JD, Wangisi J, et al. Antiretroviral prophylaxis for HIV prevention in heterosexual men and women. $\mathrm{N}$ Engl J Med. 2012;367(5):399-410.

15. Thigpen MC, Kebaabetswe PM, Paxton LA, Smith DK, Rose CE, Segolodi TM, et al. Antiretroviral preexposure prophylaxis for heterosexual HIV transmission in Botswana. N Engl J Med. 2012;367(5):423-34.

16. Van Damme L, Corneli A, Ahmed K, Agot K, Lombaard J, Kapiga S, et al. Preexposure prophylaxis for HIV infection among African women. N Engl J Med. 2012;367(5):411-22.

17. Choopanya K, Martin M, Suntharasamai P, Sangkum U, Mock PA, Leethochawalit M, et al. Antiretroviral prophylaxis for HIV infection in injecting drug users in Bangkok, Thailand (the Bangkok Tenofovir Study): a randomised, double-blind, placebo-controlled phase 3 trial. Lancet. 2013;381(9883):2083-90.

18. Marrazzo JM, Ramjee G, Richardson BA, Gomez K, Mgodi N, Nair G, et al. Tenofovir-based preexposure prophylaxis for HIV infection among African women. N Engl J Med. 2015;372(6):509-18.

19. Molina JM, Capitant C, Spire B, Pialoux G, Cotte L, Charreau I, et al. On-demand preexposure prophylaxis in men at high risk for HIV-1 infection. N Engl J Med. 2015;373(23):2237-46.

20. McCormack S, Dunn DT, Desai M, Dolling DI, Gafos M, Gilson R, et al. Pre-exposure prophylaxis to prevent the acquisition of HIV-1 infection (PROUD): effectiveness results from the pilot phase of a pragmatic open-label randomised trial. Lancet.

2016;387(10013):53-60.

21.• Mayer KH, Molina J-M, Thompson MA, Anderson PL, Mounzer KC, De Wet JJ, et al. Emtricitabine and tenofovir alafenamide $<\mathrm{em}>\mathrm{vs}</ \mathrm{em}>$ emtricitabine and tenofovir disoproxil fumarate for HIV pre-exposure prophylaxis (DISCOVER): primary results from a randomised, double-blind, multicentre, active-controlled, phase 3, non-inferiority trial. Lancet. 2020;396(10246):239-54.

Recent randomized controlled trial showing that PrEP as TAF/ FTC is superior to PrEP as TDF/FTC.

22. Margot NA, Waters JM, Miller MD. In vitro human immunodeficiency virus type 1 resistance selections with combinations of tenofovir and emtricitabine or abacavir and lamivudine. Antimicrob Agents Chemother. 2006;50(12):4087-95.

23. Grant RM, Liegler T, Defechereux P, Kashuba AD, Taylor D, Abdel-Mohsen $\mathrm{M}$, et al. Drug resistance and plasma viral RNA level after ineffective use of oral preexposure prophylaxis in women. Aids 2015;29(3):331-7.
24. Knox DC, Anderson PL, Harrigan PR, Tan DH. Multidrug-resistant HIV-1 infection despite preexposure prophylaxis. N Engl J Med. 2017;376(5):501-2.

25. Thaden JT, Gandhi M, Okochi H, Hurt CB, McKellar MS. Seroconversion on preexposure prophylaxis: a case report with segmental hair analysis for timed adherence determination. Aids. 2018;32(9):F1-f4.

One the three case reports describing PrEP failure due to transmission of HIV resistant to TDF and FTC.

26. Markowitz M, Grossman H, Anderson PL, Grant R, Gandhi M, Horng H, et al. Newly acquired infection with multidrug-resistant HIV-1 in a patient adherent to preexposure prophylaxis. J Acquir Immune Defic Syndr. 2017;76(4):e104-e6.

27. Kuritzkes DR. Preventing and managing resistance in the clinical setting. JAcquirImmuneDeficSyndr. 2003;34(Suppl 2):S103-S10.

28. Bennett B, Branson B, Delaney K, Owen M, Pentella M, Werner B. HIV testing algorithms: a status report. Silver Spring: The Association of Public Health Laboratories; 2009.

29. Cuevas JM, Geller R, Garijo R, Lopez-Aldeguer J, Sanjuan R. Extremely High Mutation Rate of HIV-1 In Vivo. PLoS Biol. 2015;13(9):e1002251.

30. Invernizzi CF, Coutsinos D, Oliveira M, Moisi D, Brenner BG, Wainberg MA. Signature nucleotide polymorphisms at positions 64 and 65 in reverse transcriptase favor the selection of the $\mathrm{k} 65 \mathrm{r}$ resistance $\mathrm{mu}-$ tation in HIV-1 subtype C. J Infect Dis. 2009;200(8):1202-6.

31. Brenner BG, Oliveira M, Doualla-Bell F, Moisi DD, Ntemgwa M, Frankel F, et al. HIV-1 subtype C viruses rapidly develop K65R resistance to tenofovir in cell culture. AIDS. 2006;20(9):F9-13.

32. Misra K, Huang J, Daskalakis DC, Udeagu CC. Impact of PREP on drug resistance and acute HIV infection, NEW YORK CITY, 2015-2017. Conference on Retroviruses and Opportunistic Infections (CROI); March 4-7 2019; Seattle; 2019. p. Abstract 107.

Study only published as an abstract showing that about $25 \%$ of patients who used PrEP before diagnosis are infected with a virus resistant to emtricitabibe

33.• Tittle V, Boffito M, McOwan A, Whitlock G. Antiretroviral resistance and management after pre-exposure prophylaxis. Lancet HIV. 2020;7(2):e84.

Summary of patients who used PrEP before diagnosis and in whom about $25 \%$ had a virus resistant to emtricitabine. The study includes an overview of the antiretroviral drug treatment given to the patients.

34. Garcia-Lerma JG, Cong ME, Mitchell J, Youngpairoj AS, Zheng Q, Masciotra S, et al. Intermittent prophylaxis with oral truvada protects macaques from rectal SHIV infection. Sci Transl Med. 2010;2(14):14ra4.

35. Saag MS, Gandhi RT, Hoy JF, Landovitz RJ, Thompson MA, Sax PE, et al. Antiretroviral Drugs for Treatment and Prevention of HIV Infection in Adults: 2020 Recommendations of the International Antiviral SocietyUSA Panel. JAMA. 2020;324(16):1651-69. 
International HIV treatment guideline that includes expert opinion and anecdotal evidence of treatment of patients in whom drug resistance due to PrEP can be found.

36. Jain V, Sucupira MC, Bacchetti P, Hartogensis W, Diaz RS, Kallas EG, et al. Differential persistence of transmitted HIV-1 drug resistance mutation classes. J Infect Dis. 2011;203(8):1174-81.

37. Wijting IEA, Lungu C, Rijnders BJA, van der Ende ME, Pham HT, Mesplede T, et al. HIV-1 resistance dynamics in patients with virologic failure to dolutegravir maintenance monotherapy. J Infect Dis. 2018;218(5):688-97.

38. Schuurman R, Nijhuis $M$, van Leeuwen R, Schipper $P$, de Jong D, Collis P, et al. Rapid changes in human immunodeficiency virus type 1 RNA load and appearance of drug-resistant virus populations in persons treated with lamivudine (3TC). JInfectDis.

1995;171(6):1411-9.

39. Nijhuis M, Schuurman R, de Jong D, van Leeuwen R, Lange J, Danner S, et al. Lamivudine-resistant human immunodeficiency virus type 1 variants $(184 \mathrm{~V})$ require multiple amino acid changes to become co-resistant to zidovudine in vivo. J Infect Dis. 1997;176(2):398-405.

40. Harrigan PR, Stone C, Griffin P, Nájera I, Bloor S, Kemp $\mathrm{S}$, et al. Resistance profile of the human immunodeficiency virus type 1 reverse transcriptase inhibitor abacavir (1592U89) after monotherapy and combination therapy. CNA2001 Investigative Group. J Infect Dis. 2000;181(3):912-20.

41. Castagna A, Danise A, Menzo S, Galli L, Gianotti N, Carini E, et al. Lamivudine monotherapy in HIV-1infected patients harbouring a lamivudine-resistant virus: a randomized pilot study (E-184V study). Aids. 2006;20(6):795-803.

42. Wolf K, Walter H, Beerenwinkel N, Keulen W, Kaiser R, Hoffmann D, et al. Tenofovir resistance and resensitization. Antimicrob Agents Chemother. 2003;47(11):3478-84.

43. Acosta RK, Willkom M, Martin R, Chang S, Wei X, Garner W, et al. Resistance analysis of bictegraviremtricitabine-tenofovir alafenamide in HIV-1 treatment-naive patients through 48 weeks. Antimicrob Agents Chemother. 2019;63(5):e02533-18.

44. Andreatta K, Willkom M, Martin R, Chang S, Wei L, Liu $\mathrm{H}$, et al. Switching to bictegravir/emtricitabine/ tenofovir alafenamide maintained HIV-1 RNA suppression in participants with archived antiretroviral resistance including M184V/I. J Antimicrob Chemother. 2019;74(12):3555-64.

45. Olearo F, Nguyen H, Bonnet F, Yerly S, Wandeler G, Stoeckle M, et al. Impact of the M184V/I mutation on the efficacy of abacavir/lamivudine/dolutegravir therapy in hiv treatment-experienced patients. Open Forum Infect Dis. 2019;6(10):ofz330.

46. Aboud M, Kaplan R, Lombaard J, Zhang F, Hidalgo JA, Mamedova E, et al. Dolutegravir versus ritonavirboosted lopinavir both with dual nucleoside reverse transcriptase inhibitor therapy in adults with HIV-1 infection in whom first-line therapy has failed
(DAWNING): an open-label, non-inferiority, phase 3b trial. Lancet Infect Dis. 2019;19(3):253-64.

47. van Wyk J, Ajana F, Bisshop F, De Wit S, Osiyemi O, Portilla Sogorb J, et al. Efficacy and safety of switching to dolutegravir/lamivudine fixed-dose 2-drug regimen vs continuing a tenofovir alafenamide-based 3- or 4drug regimen for maintenance of virologic suppression in adults living with human immunodeficiency virus type 1: phase 3, randomized, noninferiority TANGO study. Clin Infect Dis. 2020;71(8):1920-9.

48. De Miguel R, Rial-Crestelo D, Dominguez-Dominguez L, Montejano R, Esteban-Cantos A, Aranguren-Rivas P, et al. Dolutegravir plus lamivudine for maintenance of HIV viral suppression in adults with and without historical resistance to lamivudine: 48-week results of a non-randomized, pilot clinical trial (ART-PRO). EBioMedicine. 2020;55:102779.

49. Gagliardini R, Ciccullo A, Borghetti A, Maggiolo F, Bartolozzi D, Borghi V, et al. Impact of the M184V resistance mutation on virological efficacy and durability of lamivudine-based dual antiretroviral regimens as maintenance therapy in individuals with suppressed HIV-1 RNA: a cohort study. Open Forum Infect Dis. 2018;5(6):ofy113.

50. Scruggs ER, Dirks Naylor AJ. Mechanisms of zidovudine-induced mitochondrial toxicity and myopathy. Pharmacology. 2008;82(2):83-8.

51. Frentz D, Boucher CA, Assel M, De Luca A, Fabbiani M, Incardona $\mathrm{F}$, et al. Comparison of HIV-1 genotypic resistance test interpretation systems in predicting virological outcomes over time. PLoS One. 2010;5(7):e11505.

52. Hofstra LM, Sauvageot N, Albert J, Alexiev I, Garcia F, Struck D, et al. Transmission of HIV drug resistance and the predicted effect on current first-line regimens in Europe. Clin Infect Dis. 2016;62(5):655-63.

53. Margolis DA, Boffito M. Long-acting antiviral agents for HIV treatment. Curr Opin HIV AIDS. 2015;10(4):246-52.

54. Markowitz M, Grobler JA. Islatravir for the treatment and prevention of infection with the human immunodeficiency virus type 1 . Curr Opin HIV AIDS 2020;15(1):27-32.

55. Radzio-Basu J, Council O, Cong ME, Ruone S, Newton A, Wei X, et al. Drug resistance emergence in macaques administered cabotegravir long-acting for pre-exposure prophylaxis during acute SHIV infection. Nat Commun. 2019;10(1):2005.

\section{Publisher's Note}

Springer Nature remains neutral with regard to jurisdictional claims in published maps and institutional affiliations. 